\title{
Influence of Branched Polyester Chains on the Emission Behavior of Dipyridamole Molecule and Its Biosensing Ability
}

\author{
Selvaraj Nagarajan, ${ }^{\dagger} \ddagger$ Vandana Sankar, ${ }^{\ddagger}$ Kochan Sathyaseelan Bejoymohandas, ${ }^{\ddagger}$ Yongxin Duan, ${ }^{\dagger}$ \\ and Jianming Zhang* ${ }^{*}+(1)$
}

${ }^{\dagger}$ Ministry of Education/Shandong Provincial Key Laboratory of Rubber-Plastics, Qingdao University of Science \& Technology,
Qingdao City 266042, People’s Republic of China

${ }^{\ddagger}$ Materials Science and Technology Division/Agro-Processing and Natural Products Division, National Institute for Interdisciplinary Science and Technology, Thiruvananthapuram 695019, India

Supporting Information

ABSTRACT: Toward the development of the smart biosensing drug carrier, integration of dye molecules with polymeric chain has been an emerging method in recent years. In this perspective, dipyridamole (Dip)-based branched poly(L-lactide) (PLLA) and branched polycaprolactone (PCL) have been synthesized by ringopening polymerization. After polymerization, the influence of the polyester chains on the Dip emission behavior has been studied systematically in this work. Dip-PLLA has undergone $\mathrm{C}=\mathrm{O} \cdots \mathrm{N}=$ $\mathrm{C}$ interaction in ground stage, leading to intramolecular charge transfer in the excited state. Limited availability of the $\mathrm{C}=\mathrm{O}$ in PCL chains resists such interactions with Dip molecule. So, this structural availability of the $\mathrm{C}=\mathrm{O}$ group in the polymeric chains influences the color change between Dip-PLLA (green fluorescence) and DipPCL (blue fluorescence). To visualize the biosensing ability of DipPLLA and Dip-PCL, hollow microspheres have been prepared by the double-emulsion solvent evaporation method, and the prepared microspheres cells uptake has been visualized by fluorescence imaging.

\section{INTRODUCTION}

Biocompatible fluorescence dyes have become attractive materials in recent years due to their widespread application as biosensors and biomarkers due to their tracking behavior. This fluorescence dye tracking property has several advantages over traditional methods, such as radiation-free nature, high sensitivity, and specificity toward target detection with the low concentration of chromophores. ${ }^{1-4}$ The fluorescent biomarker or dye along with magnetic particles, monoclonal antibody, DNA, and drug needs to fabricate drug carriers to improve the performance. ${ }^{5}$ At the same time, multiple compounds fabrication is a complicated process. ${ }^{6}$ To simplify the process, Zhang and co-workers first introduced the multifluorescence core into mesoporous silica as a trackable drug carrier. ${ }^{7}$ The single drug carrier molecule that integrates multifunctional application is cost-effective. ${ }^{8}$

The photophysical properties of the dyes can be influenced by many factors such as temperature, ${ }^{9,10} \mathrm{pH},{ }^{11,12}$ solvent polarity, ${ }^{13}$ and physical interaction between the chromophores. ${ }^{14,15}$ Several groups demonstrated that the physical blending structure of polymer chains influences the photophysical properties of the dye. ${ }^{16,17}$ Recently, researchers have found that the covalent attachment of the dye molecule with a polymeric chain has more advantages than physical bending. Asha and co-workers reported that the covalent attachment of the oligo( $p$-phenylene vinylene)-2-diol with poly(L-lactide) (PLLA) leads to helicity transformation from the polymeric chain to oligo(phenylene vinylene). ${ }^{18,19}$ Hong's group observed that crystalline phases enhance the emission behavior, which specified as crystallization-enhanced emission with the covalent bonding of the salicylideneazine molecule with PLLA. ${ }^{20}$ Tang and co-workers ${ }^{21}$ eliminated the " $\pi-\pi$ " stacking of tetraphenylethylene (TPE) by covalent attachment of polyester chains and obtained aggregation-induced emission $^{19}$ in the solid state. Very recently, they have demonstrated the temperature-dependent on/off emission behavior of TPE end-caped polycaprolactone (PCL). ${ }^{9}$ Many interesting studies have been carried out with dye end-capped polymers, but few studies focused on the physical interaction of the polymeric chain with the dye molecules and its influence on the emission behavior. It is worth exploring the interaction science and the emission behavior between fluorescent dye and the different polymeric chains.

Therefore, in this present work, we focus on the study of the emission of Dip in different polyester environments using Dip-PLLA and Dip-PCL. Due to their excellent biodegrad-

Received: June 25, 2018

Accepted: August 15, 2018

Published: November 15, 2018 
able and biocompatible properties, both PLLA and PCL have often been considered as the ideal candidate for polymeric drug carriers. It is expected that this study will promote the role that polymeric chains play on the emission behavior of biopolymer-dye macromolecules. Parallelly, we demonstrate the fluorescence-sensing ability of the fluorescence emissive hollow microspheres of Dip-PLLA and Dip-PCL as drug carriers in the in vitro environment.

\section{RESULTS AND DISCUSSION}

2.1. Influence of the Polymer Chain Interaction with Dipyridamole on the Photophysical Properties. DipPLLA and Dip-PCL were synthesized by ring-opening polymerization using Dip as a co-initiator along with $\mathrm{Sn}(\mathrm{II}) \mathrm{Oct}_{2}$. The molecular weight $\left(M_{\mathrm{n}}\right)$ and polydispersity index of Dip-PLLA and Dip-PCL are $53 \mathrm{kDa}$ and 1.35 and $46 \mathrm{kDa}$ and 1.3 , respectively. During polymerization, the emissive Dip molecule acts as a four-arm initiator along with $\mathrm{Sn}(\mathrm{II}) \mathrm{Oct}_{2}$ and exists as an isolated entity on the branched polymer matrix. Dip is a well-studied green fluorescent compound with an emission maximum observed at $500 \mathrm{~nm}$. It exhibits a good solution-state emission efficiency with $\Phi=$ $17 \%$ (Table 1), compared to the film state $\Phi=2 \%$, which indicates that at higher concentration or in solid state, Dip molecules undergo $\pi-\pi$ stacking between the neighboring aromatic pyrimido pyrimidine rings, ${ }^{22,23}$ which leads to aggregation-caused quenching from the emissive excited state.

During polymerization, the chain rigidity of the polymeric matrix will lead to eliminating $\pi-\pi$ interactions between the neighboring Dip molecules. ${ }^{21}$ The structures of Dip, DipPLLA, and Dip-PCL are given in Figure 1a along with the photographs of Dip-PLLA and Dip-PCL polymer films under UV excitation (365 nm) and daylight. These photographs indicate the color difference of green fluorescence and blue fluorescence between Dip-PLLA and Dip-PCL in the excited state. The thin-film measurements of absorption and emission are given in Figure 1b. Even though the core molecule responsible for the fluorescence is the same, the difference in their emission color can be related to the difference in the steric situation of acceptor and the available acceptor concentration $(-\mathrm{C}=\mathrm{O})$ in the polymeric chain around the chromophore as well as the rigidity of the polymer matrix. Moreover, the differential scanning calorimetry (DSC) curves (Figure S1) for Dip-PLLA and Dip-PCL exhibit glasstransition temperatures $\left(T_{\mathrm{g}}\right.$ 's) of 60 and $-40{ }^{\circ} \mathrm{C}$, respectively. The vast difference in the $T_{\mathrm{g}}$ pointed out that the Dip-PLLA chains are much more rigid than the Dip-PCL chains. The red-shifted emission in Dip-PLLA can be attributed to the $\mathrm{C}=\mathrm{O} \cdots \mathrm{N}=\mathrm{C}$ interaction and rigid environment in the ground state and the usual process excited state with chargetransfer (CT) character, such as intramolecular charge transfer (ICT).

Intramolecular charge transfer (ICT) is a natural energy migration process, which takes place within the molecules that consist of both acceptor and donor functional groups separated in a microenvironment. Upon excitation, the charge transfer from donor to acceptor takes place in the excited state, which may produce a new charge-transfer (CT) excited state. ${ }^{24-27}$ The structure of dipyridamole consists of three different nitrogen atoms in the form of diethanolamines, piperidine rings, and aromatic pyrimido pyrimidine center. Viard et al. proved that the amino and carbonyl groups could act as an electron donor and acceptor groups, respectively. ${ }^{28}$ The

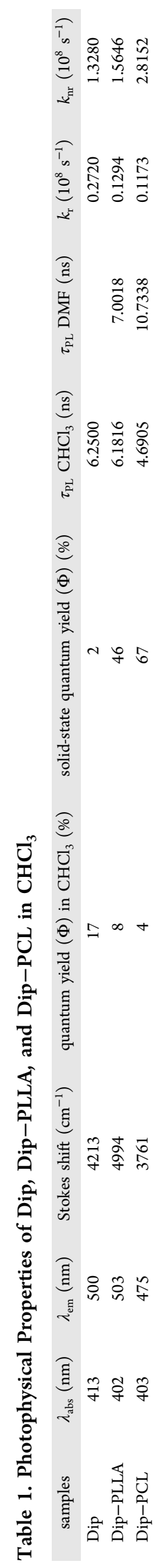


(a)

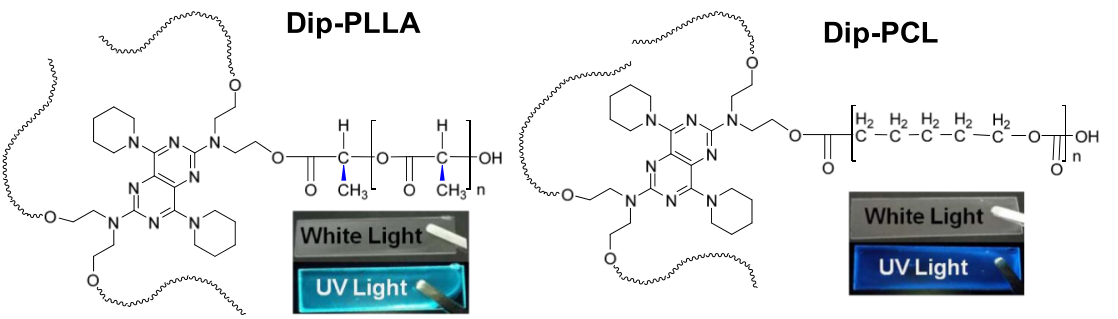

(b)

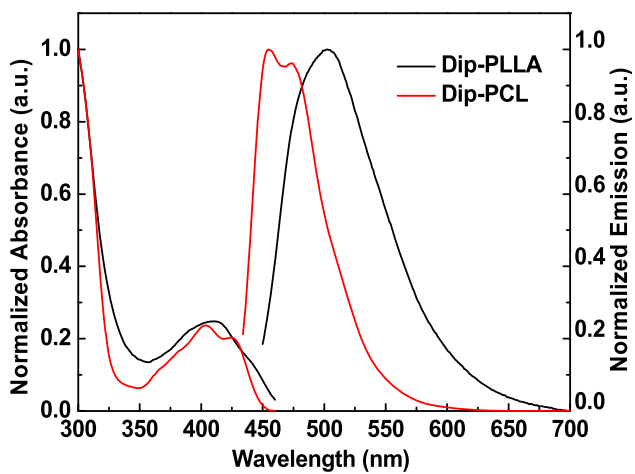

Figure 1. (a) Chemical structures of Dip-PLLA and Dip-PCL polymers and polymer-coated quartz plate in UV and daylight. (b) Excitation and emission spectra of Dip-PLLA and Dip-PCL thin films.

possibility of interaction between the aromatic pyrimido pyrimidine rings and the carbonyl groups of the ester moiety from the polymer chains cannot be neglected. The Fourier transform infrared (FTIR) spectra were recorded and compared to Dip, Dip-loaded as well as Dip-unloaded PCL and PLLA polymer films, and are shown in Figure 2. The peak

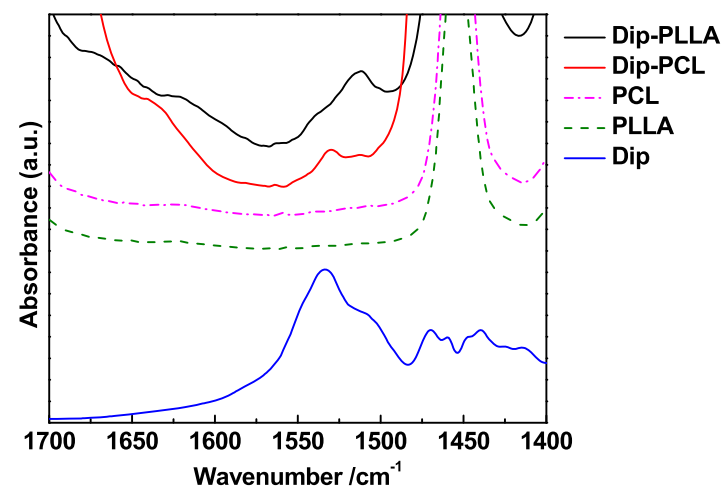

Figure 2. FTIR spectra of Dip, PCL, PLLA, Dip-PLLA, and DipPCL in the film state.

at $1533 \mathrm{~cm}^{-1}$ corresponds to the $\mathrm{N}=\mathrm{C}$ ring of the Dip. The polymers without Dip (PCL and PLLA) do not have such peaks in this region. In the case of Dip and Dip-PCL, both do not have much difference in the aromatic pyrimido pyrimidine ring-based $\mathrm{N}=\mathrm{C}$ vibrational peak $\left(1533 \mathrm{~cm}^{-1}\right)$. At the same time, in Dip-PLLA, the $\mathrm{N}=\mathrm{C}$ peak clearly shows a deviation from 1533 to $1511 \mathrm{~cm}^{-1}$ compared to Dip and Dip-PCL. The peak deviation at $1533 \mathrm{~cm}^{-1}$ confirms the interaction of $\mathrm{C}=$ $\mathrm{O} \cdots \mathrm{N}=\mathrm{C}$ in Dip-PLLA between the polymer ester chain and dipyridamole core rings. ${ }^{16,17}$ Interestingly, the long alkyl chain ester of PCL does not exhibit such interaction, due to the limited accessibility of the carbonyl groups surrounded by the Dip molecule. When Dip is integrated within the branched polymers, its emission behavior will heavily depend on the microenvironment parameters, such as acceptor concentration and molecular packing in the polymeric matrix. The absorption and emission spectra of dipyridamole, Dip-PLLA, and DipPCL are given in Figure 3. The $\lambda_{\mathrm{abs}}$ values of Dip, Dip-PLLA, and Dip-PCL are 413, 402, and $403 \mathrm{~nm}$, respectively. Dip and Dip-PCL show vibronic results of monoexponential decay with $10.73 \mathrm{~ns}$ in dimethylformamide (DMF). In the case of Dip-PLLA in DMF, results from moderate change with triexponential decay. These results indicate that the introduction of DMF in Dip-PCL alters triexponential decay into monoexponential decay. This microenvironment leads to an increase in the fluorescence intensity.

Irrespective of structural difference, both the polymers exhibit relatively high nonradiative decay rates (Table 1 ), due to the nonradiative vibrational pathways associated with the $\mathrm{CH}_{2}$ as well as $\mathrm{COO}$ bonds. Compared to Dip, the Dip-PCL absorbance exhibits a $25 \mathrm{~nm}$ blue-shifted emission maximum and both the compounds show a similarity in the vibrational progression of the emission profile. The well-resolved emission peaks confirm the lack of any charge-transfer transition in the excited state in both the compounds. Strikingly, Dip-PLLA exhibits a bathochromic shift of broad peak at $503 \mathrm{~nm}$ compared to Dip and Dip-PCL, which indicates the existence of ICT in the emission process. ${ }^{29-31}$ It is also noted that difference in the solvent polarity has no significant influence on the peak positioning of all three compounds (Figure S3), while the emission intensity enhanced with the concentration of $\mathrm{CHCl}_{3}$ solutions (Figures S4 and S5). Thus, it can be proposed that the availability of a large number of carbonyl groups in the proximity of Dip core in Dip-PLLA will lead to the formation of the weak interaction between the electrons donating the amino part of the heteroaromatic ring to the carbonyl acceptor moieties. Due to the donor-acceptor interactions, the absorption and emission spectra for Dip-PLLA appeared broad compared to the Dip and Dip-PCL. In Dip-PCL, the limited availability of the ester group (the presence of neighboring ester group only after five $\mathrm{CH}_{2}$ groups on PCL chains) prevents donor-acceptor interactions. This structural 

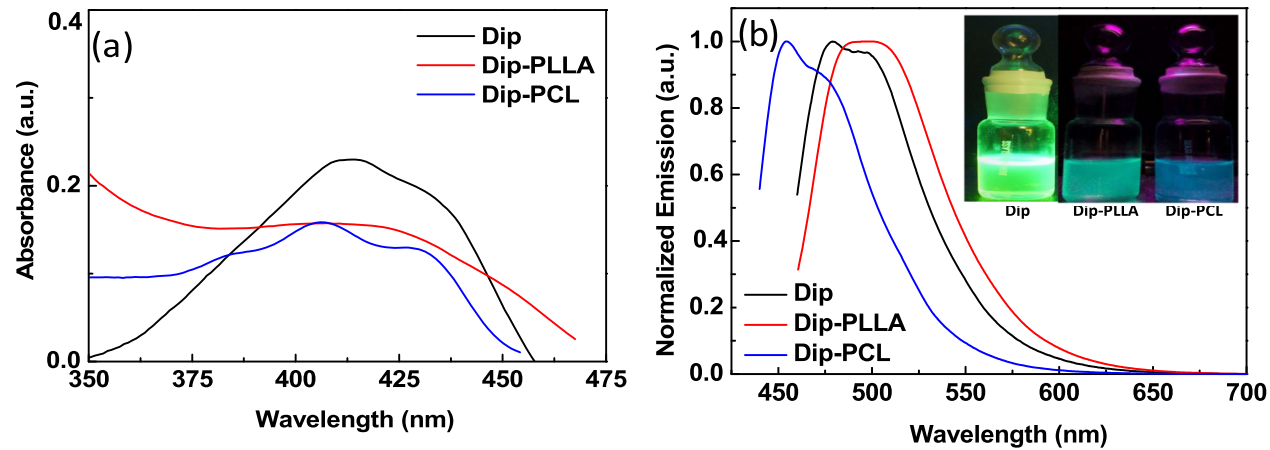

Figure 3. (a) Absorption and (b) normalized emission spectra of Dip, Dip-PLLA, and Dip-PCL in $\mathrm{CHCl}_{3}$ (inset: photographs of Dip, DipPLLA, and Dip-PCL in $\mathrm{CHCl}_{3}$ under $365 \mathrm{~nm} \mathrm{UV} \mathrm{excitation).}$
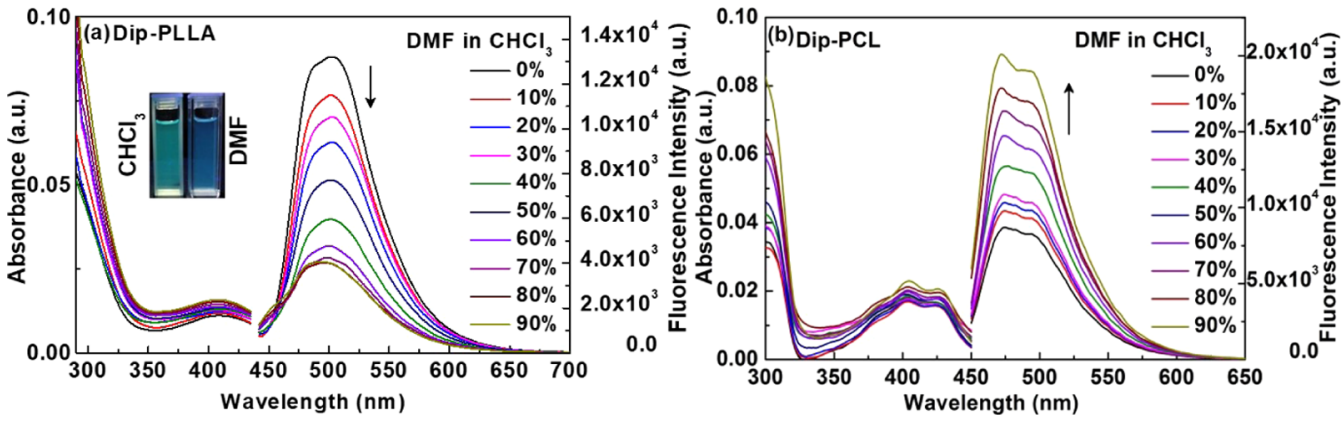

Figure 4. Excitation and emission spectra of (a) Dip-PLLA and (b) Dip-PCL in $\mathrm{CHCl}_{3}$ with different percentages of DMF.
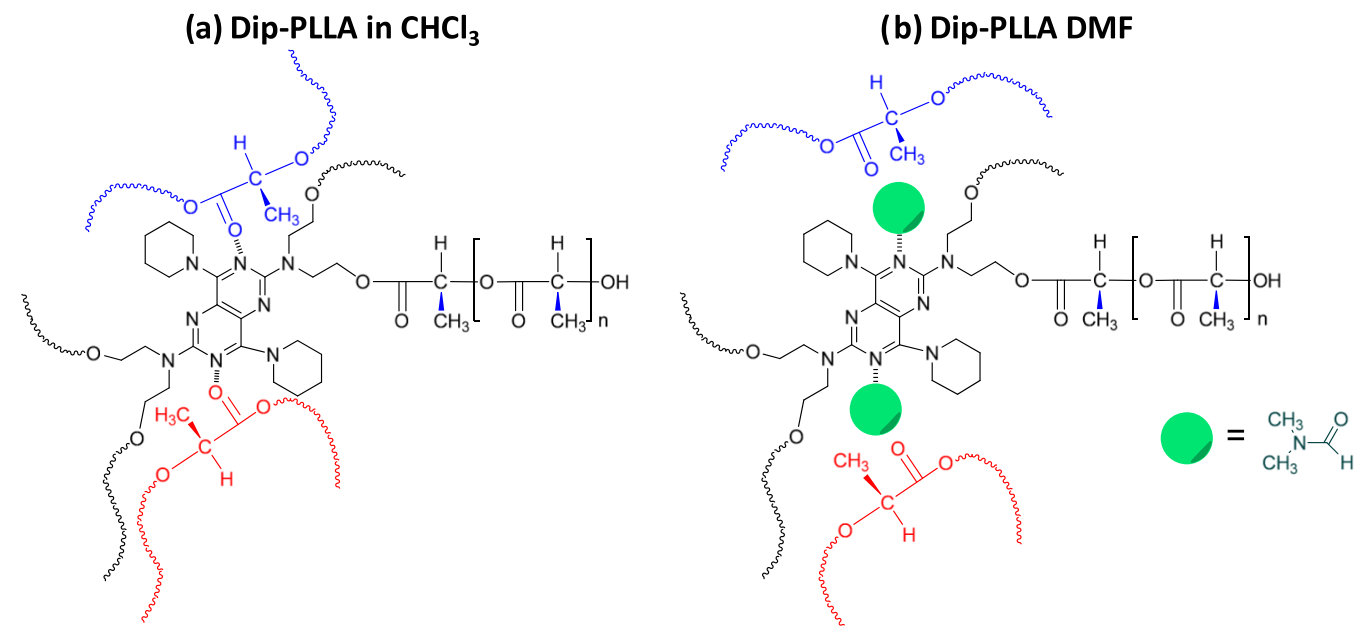

Figure 5. (a) Inter- and intra-interaction of $\mathrm{C}=\mathrm{O} \cdots \mathrm{N}=\mathrm{C}$ carbonyl group with pyrimido pyrimidine ring and (b) elimination of $\mathrm{C}=\mathrm{O} \cdots \mathrm{N}=\mathrm{C}$ interaction in the presence of DMF solvent.

construction offers the Dip chromophore in Dip-PCL to emit in the vibronic state even at low to high concentrations. The weak bonding in Dip-PLLA has led to a larger Stokes shift of $4994 \mathrm{~cm}^{-1}$ compared to Dip-PCL $\left(3761 \mathrm{~cm}^{-1}\right)$, and this observation further supports the above speculations. ${ }^{32-34}$ Figure S5 shows the absorption and emission spectra of Dip by adjusting the polarity with the different composition of $\mathrm{CHCl}_{3}$ and DMF. It is interesting to note that increasing the concentration of DMF has structured the emission profile with well-resolved vibronic peaks. The same observation is also noted for Dip-PCL (Figure 4b). While in the case of DipPLLA, increasing DMF concentration blue-shifted the emission maximum from 502 to $491 \mathrm{~nm}$, parallelly, emission peaks are also getting a resolved structure along with the quenching of luminescence (Figure 4a). This behavior is quite interesting from Dip-PCL, and the steric situation of the Dip-PLLA allows the $\mathrm{C}=\mathrm{O} \cdots \mathrm{N}=\mathrm{C}$ bonding interactions. This complex $\mathrm{C}=\mathrm{O} \cdots \mathrm{N}=\mathrm{C}$ interaction of Dip-PLLA was significantly affected by the aprotic solvent. The environmental effect of the chromophore is complex, and the solvent polarity cannot relate to a single theory. ${ }^{24,35}$ From the results, we speculate that the ICT state is stabilized by the $\mathrm{C}=\mathrm{O} \cdots \mathrm{N}=\mathrm{C}$ interaction between the emissive Dip core and $\mathrm{C}=\mathrm{O}$ group of the polymeric chain, and when DMF is added, the $\mathrm{C}=\mathrm{O}$ group of the DMF replaces the $\mathrm{C}=\mathrm{O} \cdots \mathrm{N}=\mathrm{C}$ interaction between the polymer chain and Dip molecules, as can be seen from the schematic representation in Figure $5 .{ }^{36}$ As a result of this, the molecular rigidity decreases, which leads to more 


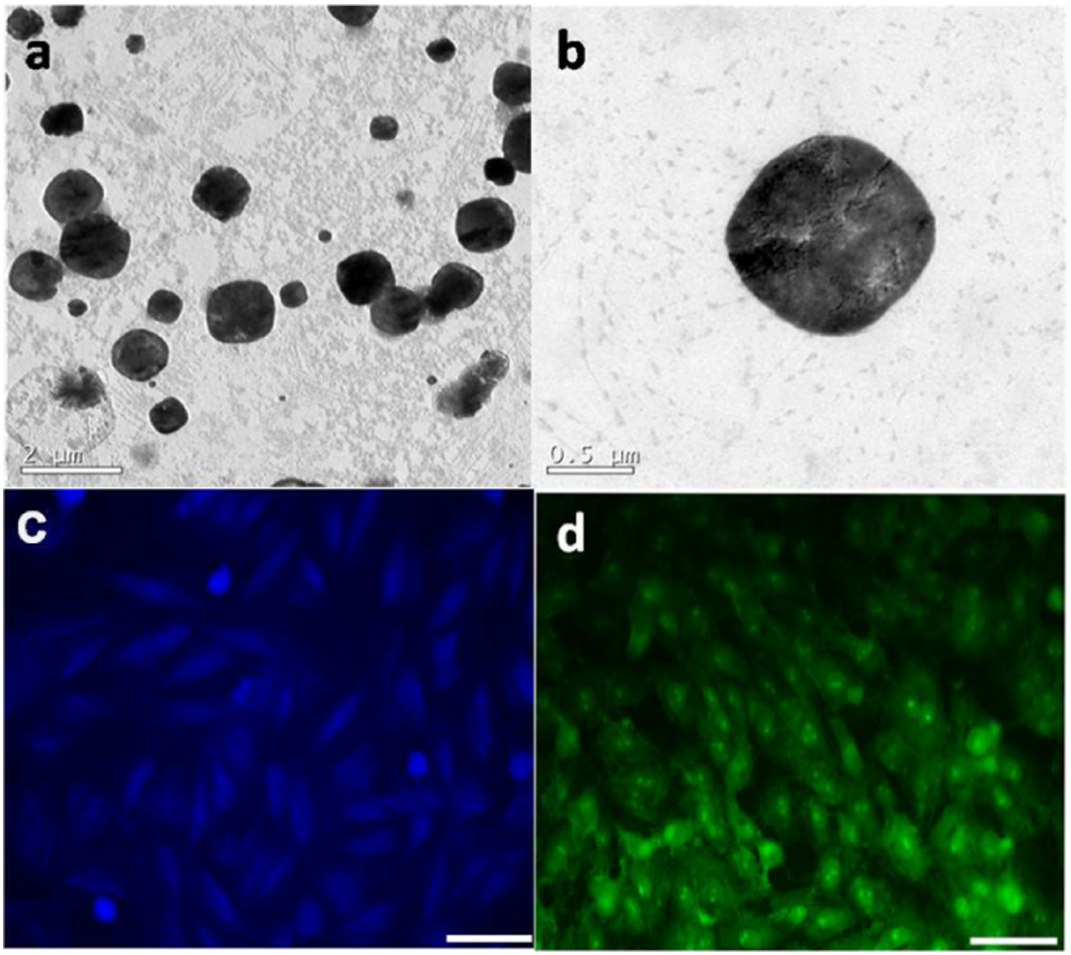

Figure 6. (a, b) TEM images of Dip-PLLA microspheres. Confocal microscopy images of cells: (c) Dip-PCL and (d) Dip-PLLA, and the corresponding fluorescence images of $(\mathrm{d}) \mathrm{H} 9 \mathrm{c} 2$ cell lines incubated with the polymer at a concentration of $2.5 \mu \mathrm{g} / \mathrm{mL}\left(\lambda_{\mathrm{ex}}=404 \mathrm{~nm}\right.$; scale bar $=$ $100 \mu \mathrm{m})$.

nonradiative decay pathways. On the other hand, the aromatic pyrimido pyrimidine centers attract to each other and may form $\mathrm{H}$-aggregation, which will result in the blue shift of the emission profile. In the case of Dip-PCL, the increasing concentration of DMF enhances the emission intensity, and emission profiles were observed as vibrationally well resolved.

2.2. Hollow Microsphere: In Vitro Fluorescence Sensing. Hollow microspheres were fabricated with single polymer Dip-PLLA or Dip-PCL by the double-emulsion method. The microspheres diameter is around $1-2 \mu \mathrm{m}$, as observed from the transmission electron microscopy (TEM) images in Figure 6. These microspheres were filtered three times using a syringe filter to eliminate large particles. The branched star-shaped architecture color change was observed by confocal microscopy not making much difference in building block to assemble comparative to Dip-PLLA and Dip-PCL in Figure S7. This Dip-based polyester has good biocompatibility with potential anticoagulation and angiogenesis in the living host, which was evident from our earlier investigations. $^{37}$ In this study, H9c2 cell lines were incubated with the microspheres in an aqueous medium. The intracellular uptakes of Dip-PLLA and Dip-PCL microsphere could be visualized after $1 \mathrm{~h}$ by live cell imaging, based on the intrinsic fluorescence property of microsphere. The blue fluorescence could visualize the Dip-PCL uptake in the cells, and it spread throughout the cell cytoplasm. Dip-PLLA microsphere uptake could be observed as a localized green fluorescence in the certain region of the cells. The fluorescence intensity of DipPLLA increased with increase in the concentration of microsphere, as shown in Figure S8. These Dip-based polymer microspheres are prepared from the single molecule, so dye leakage will not occur like multiple additives spheres. ${ }^{4,5}$ On the other hand, the hydrophilicity of the drug carrier plays an important role to permeate through the cell membrane via endocytosis process. This cell permeation of a drug carrier with the size of $1-2 \mu \mathrm{m}$ can be obtained by coating the particle surface with hydrophilic vitamin E or PEG-1000. ${ }^{38-41}$ But in the case of Dip-PLLA- and Dip-PCL-based spheres without any post-treatment, it can be easily permeated via cell wall, which is due to more hydroxyl terminal groups in the branching system, which enhanced the hydrophilicity of the polymer, leading to cell adherence with more biological activity. ${ }^{37}$ The glass transition of the polymer does not have direct influence on the bioactivities. Integrating, the synergetic effect of the additives in a single molecule as a multifunctionalized material will simplify the fabrication of drug carrier and reduce the cost. $6,8,42,43$

\section{CONCLUSIONS}

The influence of the polyester chains on the emission of Dip molecule was studied using Dip-PLLA and Dip-PCL. The interaction of $\mathrm{C}=\mathrm{O} \cdots \mathrm{N}=\mathrm{C}$ bonding in between PLLA chains and Dip has been identified in Dip-PLLA by FTIR spectroscopy. This ground-state $\mathrm{C}=\mathrm{O} \cdots \mathrm{N}=\mathrm{C}$ bonding leads to intramolecular charge transfer (ICT) in the excited state in Dip-PLLA. In Dip-PCL, the availability of the -COO group is limited compared to Dip-PLLA, which resists the $\mathrm{C}=\mathrm{O} \cdots$ $\mathrm{N}=\mathrm{C}$ interaction with Dip, and it exhibits vibronic progression in the emission profile. The addition of DMF damages the interaction between the Dip emissive core and $\mathrm{C}=\mathrm{O}$ groups of the polymer chain in PLLA. It leads to the change of color from green fluorescence to blue fluorescence in Dip-PLLA. It identified that the interaction of $\mathrm{C}=\mathrm{O} \cdots \mathrm{N}=\mathrm{C}$ is the key factor for color change in Dip-PLLA. Fluorescencesensible hollow microspheres of Dip-PLLA and Dip-PCL were fabricated by the double-emulsion method with micro- 
encapsulation technique, and the cell uptake of Dip-based spheres was visualized via fluorescence images under in vitro conditions.

\section{EXPERIMENTAL SECTION}

4.1. Materials. Dipyridamole-based PLLA and dipyridamole-based PCL were synthesized as described in our previous report. ${ }^{37}{ }^{1} \mathrm{H}$ NMR spectrum of Dip-PCL is shown in Figure S9. Poly(vinyl alcohol) (PVA) $\left(M_{\mathrm{w}}=89-98 \mathrm{kDa}\right)$ and dipyridamole were purchased from Sigma-Aldrich. Highperformance liquid chromatography-grade dichloromethane (DCM), DMF, toluene, and chloroform were purchased from Mark Chemicals.

4.2. Microsphere Preparation. Polymer (100 mg) was dissolved in $10 \mathrm{~mL}$ of DCM. The primary emulsion of $2 \%$ of PVA was dissolved in hot water $\left(70{ }^{\circ} \mathrm{C}\right)$ and cooled down to room temperature. PVA solution $(10 \mathrm{~mL})$ was mixed with the polymer solution, and the mixture was sonicated for $10 \mathrm{~min}$ in the ice bath. PVA solution $(15 \mathrm{~mL})$ was added and sonicated for $20 \mathrm{~min}$; then, the mixture was stirred to evaporate the DCM in the solution and then centrifuged at $10000 \mathrm{rpm}$ and filtered with $0.5 \mu \mathrm{m}$ fillers.

4.3. Cell Line Maintenance. $\mathrm{H} 9 \mathrm{c} 2$ cell lines (rat embryonic cardiomyoblasts) obtained from American Type Culture Collection (ATCC). For the maintenance of cell lines, Dulbecco's modified Eagle's medium (DMEM) (SigmaAldrich) containing $10 \%$ fetal bovine serum (Gibco), antibiotics $(100 \mathrm{U} / \mathrm{mL}$ penicillin and $100 \mu \mathrm{g} / \mathrm{mL}$ streptomycin), and amphotericin $(0.25 \mu \mathrm{g} / \mathrm{mL})$ (HiMedia) was employed. The cells were maintained in cell culture flasks in $\mathrm{CO}_{2}$ incubators at $37{ }^{\circ} \mathrm{C}$ with $5 \% \mathrm{CO}_{2}$ in air and $99 \%$ humidity. Passaging of cells when confluent was carried out using $0.25 \%$ trypsin and $0.02 \%$ ethylenediaminetetraacetic acid (HiMedia) in phosphate-buffered saline.

4.4. Cellular Uptake Studies of Branched Polymers by Fluorescence Imaging. Cellular uptake studies of branched polymers were carried out by fluorescence imaging of adherent cells. The cells were seeded at a density of $5 \times 10^{3}$ cells/well of 96-well black plates (BD Biosciences) for the purpose. After 36 $\mathrm{h}$ from seeding, the cells were first washed twice with Hanks' balanced salt solution (HBSS) to remove DMEM. Then, the cells were incubated with different concentrations of branched polymers in HBSS for $1 \mathrm{~h}$. Subsequently, the cells were washed thrice with HBSS and subjected to live cell imaging. Images were collected by the high-content spinning disk facility (BD Pathway 855; BD Biosciences) using AttoVision 1.5.3 software. For imaging cellular uptake of SSPLLA, $488 / 10 \mathrm{~nm}$ excitation filter and $515 \mathrm{~nm}$ long pass (LP) emission filter were used. For System Support Package Components List (SSPCL), 360/10 $\mathrm{nm}$ excitation filter and $420 \mathrm{~nm}$ LP emission filter were used. Imaging was done using a high-content spinning disk facility from $\mathrm{BD}$ Biosciences. It is a fluorescence microscope (BD Pathway 855) connected to an image analysis package (AttoVision 1.5.3 software).

4.5. Measurement of Cell Fluorescence Intensities of Branched Polymers. Fluorescence intensity measurements were also carried out in adherent cells. The procedure adopted was the same as that of the imaging protocol. For the analyses, a multimode reader (BioTek) was used. The cells were seeded at a density of $5 \times 10^{3}$ cells/well of 96-well black plates (BD Biosciences) for this purpose. After $36 \mathrm{~h}$ from seeding, the cells were first washed twice with Hanks' balanced salt solution (HBSS). Then, the cells were incubated with different concentrations of branched polymers in HBSS for $1 \mathrm{~h}$. Subsequently, the cells were washed thrice with HBSS and subjected to a fluorimeter using a fluorescence microplate reader (Model: Synergy-4 Multimode reader, BioTek).

4.6. Instruments. Fourier transform infrared (FTIR) spectra were measured in a Bruker tensor 27 FTIR spectrometer with a resolution of $2 \mathrm{~cm}^{-1}$. The samples of Dip, Dip-PCL, and Dip-PLLA in $\mathrm{CH}_{3} \mathrm{Cl}$ solution were dropcast on $\mathrm{KBr}$ pellet, and the films were dried in a vacuum oven. The UV spectra of dipyridamole, Dip-PLLA, and Dip-PCL were measured in solution state using Shimadzu model UV3101PC-UV-NIR spectrophotometers. The emission spectra quantum yields were recorded in a SPEX-Fluorolog-3 FL3-221 spectrofluorimeter. The relative quantum yield and absolute quantum yield were obtained by a standard procedure and are given in the Supporting Information. Scanning electron microscopy (SEM) images were obtained from a Zeiss EVO 18 cryo SEM Special Edn with a variable pressure detector working at $20-30 \mathrm{kV}$. Transmission electron microscopy (TEM) was performed in an FEI TECNAI 30 G2 S-TWIN microscope at an accelerating voltage of $100 \mathrm{kV}$. The lifetimes of Dip, Dip-PLLA, and Dip-PCL were measured on an Edinburgh FLS-980 Spectrometer with an EPL-375 optical laser.

\section{ASSOCIATED CONTENT}

\section{Supporting Information}

The Supporting Information is available free of charge on the ACS Publications website at DOI: 10.1021/acsomega.8b01436.

DSC thermograms of Dip-PLLA and Dip-PCL (Figure S1); emission spectra of (a) Dip, (b) Dip-PLLA, and (c) Dip-PCL by changing the different polarity of solvents: toluene $<\mathrm{CHCl}_{3}<$ tetrahydrofuran $<$ dimethyl sulfoxide $<$ acetonitrile with the polarity indices of $2.4<$ $2.7<4<5.8<7.2$, respectively (Figure S2); absorption and emission spectra of Dip with different concentrations $\left(1 \times 10^{-2}-6 \times 10^{-2} \mathrm{~mol} / \mathrm{L}\right)$ (Figure S3); absorption and emission spectra of (a) Dip-PLLA and (b) Dip-PCL in $\mathrm{CHCl}_{3}$ with concentration of $1.0 \times$ $10^{-5}-7 \times 10^{-5} \mathrm{~mol} / \mathrm{L}$ (Figure S4); excitation and emission spectra dipyridamole in $\mathrm{CHCl}_{3}$ with different concentrations of DMF (Figure S5); fluorescence lifetime of the Dip-PCL and Dip-PLLA (Figure S6); confocal microscopy images of the microspheres of (a) Dip-PLLA and (b) Dip-PCL and the corresponding SEM images (c) and (d) (scale bar $=1 \mu \mathrm{m}$ ) (Figure S7); relative fluorescence intensities of Dip-PLLA and Dip-PCL spheres with respect to concentration (Figure S8); ${ }^{1} \mathrm{H}$ NMR spectra of SSPCL (Figure S9); and wideangle X-ray scattering plot of Dip-PLLA and Dip-PCL (Figure S10) (PDF)

\section{AUTHOR INFORMATION}

\section{Corresponding Author}

*E-mail: zjm@qust.edu.cn. Tel: +86 532 84022604. Fax: +86 53284022791 (J.Z.).

ORCID $\odot$

Selvaraj Nagarajan: 0000-0001-6707-3165

Kochan Sathyaseelan Bejoymohandas: 0000-0003-3388-4941 Jianming Zhang: 0000-0002-0252-4516 


\section{Notes}

The authors declare no competing financial interest.

\section{ACKNOWLEDGMENTS}

The authors acknowledge the financial support from Taishan Mountain Scholar Foundation (TS20081120 and tshw20110510).

\section{REFERENCES}

(1) Huis in 't Veld, R.; Storm, G.; Hennink, W. E.; Kiessling, F.; Lammers, T. Macromolecular Nanotheranostics for Multimodal Anticancer Therapy. Nanoscale 2011, 3, 4022.

(2) Li, X.; Liu, H.; Sun, X.; Bi, G.; Zhang, G. Highly Fluorescent Dye-Aggregate-Enhanced Energy-Transfer Nanoparticles for Neuronal Cell Imaging. Adv. Opt. Mater. 2013, 1, 549-553.

(3) Klemm, D.; Kramer, F.; Moritz, S.; Lindström, T.; Ankerfors, M.; Gray, D.; Dorris, A. Cellulose Nanocrystals: Chemistry, SelfAssembly, and Applications. Angew. Chem., Int. Ed. 2011, 50, 54385466.

(4) Larson, N.; Ghandehari, H. Polymeric Conjugates for Drug Delivery. Chem. Mater. 2012, 24, 840-853.

(5) Janib, S. M.; Moses, A. S.; MacKay, J. A. Imaging and Drug Delivery Using Theranostic Nanoparticles. Adv. Drug Delivery Rev. 2010, 62, 1052-1063.

(6) Lei, J.; Wang, L.; Zhang, J. Superbright Multifluorescent Core Shell Mesoporous Nanospheres as Trackable Transport Carrier for Drug. ACS Nano 2011, 5, 3447-3455.

(7) Zhang, Z.; Wang, L.; Wang, J.; Jiang, X.; Li, X.; Hu, Z.; Ji, Y.; Wu, X.; Chen, C. Mesoporous Silica Coated Gold Nanorods as a Light Mediated Multifunctional Theranostic Platform for Cancer Treatment. Adv. Mater. 2012, 24, 1418-1423.

(8) Nanomedicine: Multiplying the Possibilities NPG Asia Mater. 2011, https://www.nature.com/articles/am2011138. DOI: 10.1038/ asiamat.2011.119.

(9) Liang, G.; Wu, J.; Gao, H.; Wu, Q.; Lu, J.; Zhu, F.; Tang, B. Z. General Platform for Remarkably Thermoresponsive Fluorescent Polymers with Memory Function. ACS Macro Lett. 2016, 5, 909-914.

(10) Mao, X. L.; Shannon, M. A.; Fernandez, A. J.; Russo, R. E. Temperature and Emission Spatial Profiles of Laser-Induced Plasmas during Ablation Using Time-Integrated Emission Spectroscopy. Appl. Spectrosc. 1995, 49, 1054-1062.

(11) Tantama, M.; Hung, Y.; Yellen, G. Imaging Intracellular $\{\mathrm{pH}\}$ in Live Cells with a Genetically Encoded Red Fluorescent Protein Sensor. J. Am. Chem. Soc. 2011, 133, 10034-10037.

(12) Cai, Y.; Gui, C.; Samedov, K.; Su, H.; Gu, X.; Li, S.; Luo, W.; Sung, H. H. Y.; Lam, J. W. Y.; Kwok, R. T. K.; et al. An Acidic PH Independent Piperazine-TPE AIEgen as a Unique Bioprobe for Lysosome Tracing. Chem. Sci. 2017, 8, 7593-7603.

(13) Haidekker, M. A.; Brady, T. P.; Lichlyter, D.; Theodorakis, E. A. Effects of Solvent Polarity and Solvent Viscosity on the Fluorescent Properties of Molecular Rotors and Related Probes. Bioorg. Chem. 2005, 33, 415-425.

(14) Hunter, C. A.; Sanders, J. K. M. The Nature of .Pi.-.Pi. Interactions. J. Am. Chem. Soc. 1990, 112, 5525-5534.

(15) Li, Q.; Li, Z. The Strong Light-Emission Materials in the Aggregated State: What Happens from a Single Molecule to the Collective Group. Adv. Sci. 2017, 4, No. 1600484.

(16) Beten, D. B.; Gelbcke, M.; Diallo, B.; Moes, A. J. Interaction between Dipyridamole and Eudragit-S. Int. J. Pharm. 1992, 88, 3137.

(17) Baghel, S.; Cathcart, H.; O’Reilly, N. J. Theoretical and Experimental Investigation of Drug-Polymer Interaction and Miscibility and Its Impact on Drug Supersaturation in Aqueous Medium. Eur. J. Pharm. Biopharm. 2016, 107, 16-31.

(18) Nisha, S. K.; Asha, S. K. Chiral Poly(l-Lactic Acid) Driven Helical Self-Assembly of Oligo(p-Phenylenevinylene). J. Mater. Chem. C 2014, 2, 2051.
(19) Cai, Y.; Du, L.; Samedov, K.; Gu, X.; Qi, F.; Sung, H. H. Y.; Patrick, B. O.; Yan, Z.; Jiang, X.; Zhang, H.; et al. Deciphering the Working Mechanism of Aggregation-Induced Emission of Tetraphenylethylene Derivatives by Ultrafast Spectroscopy. Chem. Sci. 2018, 9, $4662-4670$

(20) Hsiao, T.-S.; Huang, P.-C.; Lin, L.-Y.; Yang, D.-J.; Hong, J.-L. Crystallization-Promoted Emission Enhancement of Poly ( $\langle$ scp $\rangle 1</$ Scp $\rangle$-Lactide) Containing a Fluorescent Salicylideneazine Center with Aggregation-Enhanced Emission Properties. Polym. Chem. 2015, $6,2264-2273$.

(21) Zhao, W.; Li, C.; Liu, B.; Wang, X.; Li, P.; Wang, Y.; Wu, C.; Yao, C.; Tang, T.; Liu, X.; et al. A New Strategy to Access Polymers with Aggregation-Induced Emission Characteristics. Macromolecules 2014, 47, 5586-5594.

(22) Luger, P.; Roch, J. Structure and superstructure of dipyridamole, 2,2',2",2"'-(4,8-dipiperidinopyrimido[5,4-d]pyrimidine2,6-diyldinitrilo)tetraethanol, $\mathrm{C}_{24} \mathrm{H}_{40} \mathrm{~N}_{8} \mathrm{O}_{4}$. Acta Crystallogr., Sect. C: Cryst. Struct. Commun. 1983, 39, 1454-1458.

(23) Grimme, S. Do Special Noncovalent $\pi$ - $\pi$ Stacking Interactions Really Exist? Angew. Chem., Int. Ed. 2008, 47, 3430-3434.

(24) Grabowski, Z. R.; Rotkiewicz, K.; Rettig, W. Structural Changes Accompanying Intramolecular Electron Transfer: Focuson Twisted Intramolecular Charge-Transfer States and Structures. Chem. Rev. 2003, 103, 3899-4032.

(25) Rettig, W. Charge Separation in Excited-States of Decoupled Systems - Tict Compounds and Implications Regarding the Development of New Laser-Dyes and the Primary Processes of Vision and Photosynthesis. Angew. Chem., Int. Ed. Engl. 1986, 25, 971-988.

(26) Mallick, A.; Purkayastha, P.; Chattopadhyay, N. Photoprocesses of Excited Molecules in Confined Liquid Environments: An Overview. J. Photochem. Photobiol., C 2007, 8, 109-127.

(27) Rettig, W. Photoinduced Charge Separation via Twisted Intramolecular Charge Transfer States. Top. Curr. Chem. 1994, 169, 253-299.

(28) Viard, M.; Gallay, J.; Vincent, M.; Meyer, O.; Robert, B.; Paternostre, M. Laurdan Solvatochromism: Solvent Dielectric Relaxation and Intramolecular Excited-State Reaction. Biophys. J. 1997, 73, 2221-2234.

(29) Cazeau-Dubroca, C.; Peirigua, A.; Brahim, M. B.; Nouchi, G.; Cazeau, P. Specific Hydrogen Bonding Interaction versus the "Free Volume" Model for TICT Molecules in Polymeric Matrices. Chem. Phys. Lett. 1989, 157, 393-397.

(30) Cazeau-Dubroca, C.; Nouchi, G.; Ben Brahim, M.; Pesquer, M.; Grose, D.; Cazeau, P. Dual Fluorescence of 4-N,N-Dimethylaminopyridine. Role of Hydrogen-Bonded Complex in the Ground State. J. Photochem. Photobiol., A 1994, 80, 125-133.

(31) Cazeau-Dubroca, C.; Peirigua, A.; Lyazidi, S. A.; Nouchi, G.; Cazeau, P.; Lapouyade, R. Tict Fluorescence in Rigid Matrices: $\alpha$ Delayed Fluorescence. Chem. Phys. Lett. 1986, 124, 110-115.

(32) Rosenberg, H. M.; Eimutis, E. Solvent shifts in electronic spectra-I. Stokes shift in a series of homologous aromatic amines. Spectrochim. Acta 1966, 2, 1751-1757.

(33) Werner, T. C.; Hoffman, R. M. Relation between an Excited State Geometry Change and the Solvent Dependence of 9-Methyl Anthroate Fluorescence. J. Phys. Chem. 1973, 77, 1611-1615.

(34) Werner, T. C.; Hercules, D. M. Fluorescence of 9-Anthroic Acid and Its Esters. Environmental Effects on Excited-State Behavior. J. Phys. Chem. 1969, 73, 2005-2011.

(35) Declemy, A.; Rulliere, C.; Kottis, P. Picosecond HydrogenBond Formation in Excited States: The Case of an Intramolecular Charge-Transfer (ICT) State. Chem. Phys. Lett. 1983, 101, 401-406.

(36) Chipem, F. A. S.; Mishra, A.; Krishnamoorthy, G. The Role of Hydrogen Bonding in Excited State Intramolecular Charge Transfer. Phys. Chem. Chem. Phys. 2012, 14, 8775.

(37) Nagarajan, S.; Kiran, M. S.; Tsibouklis, J.; Reddy, B. S. R. Multifunctional Star-Shaped Polylactic Acid Implants for Use in Angioplasty. J. Mater. Chem. B 2014, 2, 6549-6559. 
(38) Mu, L.; Feng, S. S. PLGA/TPGS Nanoparticles for Controlled Release of Paclitaxel: Effects of the Emulsifier and Drug Loading Ratio. Pharm. Res. 2003, 20, 1864-1872.

(39) Mu, L.; Feng, S. S. Vitamin E TPGS Used as Emulsifier in the Solvent Evaporation/Extraction Technique for Fabrication of Polymeric Nanospheres for Controlled Release of Paclitaxel (Taxol??). J. Controlled Release 2002, 80, 129-144.

(40) Mu, L.; Feng, S. S. Fabrication, Characterization and in Vitro Release of Paclitaxel (Taxol) Loaded Poly(Lactic-Co-Glycolic Acid) Microspheres Prepared by Spray Drying Technique with Lipid/ Cholesterol Emulsifiers. J. Controlled Release 2001, 76, 239-254.

(41) Feng, S.-S.; Huang, G. Effects of Emulsifiers on the Controlled Release of Paclitaxel (Taxol) from Nanospheres of Biodegradable Polymers. J. Controlled Release 2001, 71, 53-69.

(42) Mu, L.; Feng, S. S. A Novel Controlled Release Formulation for the Anticancer Drug Paclitaxel (Taxol((R))): PLGA Nanoparticles Containing Vitamin E TPGS. J. Controlled Release 2003, 86, 33-48.

(43) Liu, X.; Jin, X.; Ma, P. X. Nanofibrous Hollow Microspheres Self-Assembled from Star-Shaped Polymers as Injectable Cell Carriers for Knee Repair. Nat. Mater. 2011, 10, 398-406. 